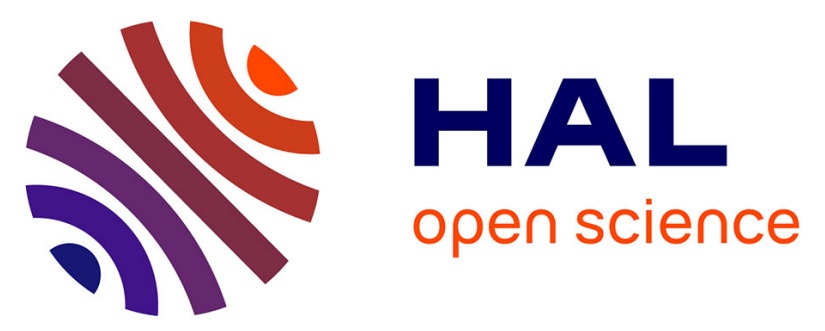

\title{
Intraoperative acceleration measurements to quantify improvement in tremor during deep brain stimulation surgery
}

Ashesh Shah, Jerome Coste, Jean-Jacques Lemaire, Ethan Taub, W. M. Michael Schüpbach, Claudio Pollo, Erik Schkommodau, Raphael Guzman, Simone Hemm-Ode

\section{To cite this version:}

Ashesh Shah, Jerome Coste, Jean-Jacques Lemaire, Ethan Taub, W. M. Michael Schüpbach, et al.. Intraoperative acceleration measurements to quantify improvement in tremor during deep brain stimulation surgery. Medical and Biological Engineering and Computing, 2017, 55 (5), pp.845 - 858. 10.1007/s11517-016-1559-9 . hal-01580256

\section{HAL Id: hal-01580256 \\ https://hal.uca.fr/hal-01580256}

Submitted on 1 Sep 2017

HAL is a multi-disciplinary open access archive for the deposit and dissemination of scientific research documents, whether they are published or not. The documents may come from teaching and research institutions in France or abroad, or from public or private research centers.
L'archive ouverte pluridisciplinaire HAL, est destinée au dépôt et à la diffusion de documents scientifiques de niveau recherche, publiés ou non, émanant des établissements d'enseignement et de recherche français ou étrangers, des laboratoires publics ou privés. 


\title{
Intraoperative acceleration measurements to quantify improvement in tremor during deep brain stimulation surgery
}

\author{
Ashesh Shah ${ }^{1}$, Jérôme Coste ${ }^{2,3}$, Jean-Jacques Lemaire ${ }^{2,3}$, Ethan Taub ${ }^{4}$, W. M. Michael \\ Schüpbach $^{5,6}$, Claudio Pollo ${ }^{7}$, Erik Schkommodau ${ }^{1}$, Raphael Guzman ${ }^{4}$, Simone Hemm-Ode ${ }^{1, *}$
}

1. Institute for Medical and Analytical Technologies, University of Applied Sciences and Arts Northwestern Switzerland, Muttenz, Switzerland

2. Image-Guided Clinical Neuroscience and Connectomics (EA 7282), Université Clermont Auvergne, ClermontFerrand, France

3. Service de Neurochirurgie, CHU Clermont-Ferrand, Clermont-Ferrand, France

4. Departments of Neurosurgery and Biomedicine, University of Basel, Basel, Switzerland

5. Department of Neurology, University Hospital Bern and University of Bern, Bern, Switzerland

6. Assistance Publique Hôpitaux de Paris, Institut National de Santé et en Recherche Médicale, Institut du Cerveau et de la Moelle Epinière, Centre d'Investigation Clinique 1422, Département de Neurologie, Hôpital Pitié-Salpêtrière, Paris, France

7. Department of Neurosurgery, University Hospital Bern, Bern, Switzerland

*. Corresponding author: Simone Hemm-Ode, simone.hemm@ fhnw.ch

\begin{abstract}
Deep brain stimulation (DBS) surgery is extensively used in the treatment of movement disorders. Nevertheless, methods to evaluate the clinical response during intraoperative stimulation tests to identify the optimal position for the implantation of the chronic DBS lead remain subjective. In this paper, we describe a new, versatile method for quantitative intraoperative evaluation of improvement in tremor with an acceleration sensor that is mounted on the patient's wrist during surgery. At each anatomical test position, the improvement in tremor compared to the initial tremor is estimated on the basis of extracted outcome measures. This method was tested on 15 tremor patients undergoing DBS surgery in two centers. Data from 359 stimulation tests were acquired. Our results suggest that accelerometric evaluation detects tremor changes more sensitively than subjective visual ratings. The effective stimulation current amplitudes identified from the quantitative data $(1.1 \pm 0.8 \mathrm{~mA})$ are lower than those identified by visual evaluation $(1.7 \pm 0.8 \mathrm{~mA})$ for similar improvement in tremor. Additionally, if these data had been used to choose the chronic implant position of the DBS lead, 15 of the 26 choices would have been different. These results show that our method of accelerometric evaluation can potentially improve DBS targeting.
\end{abstract}

Keywords Deep brain stimulation, Intraoperative monitoring, Acceleration, Tremor, Parkinson's disease, Essential tremor 


\section{Introduction}

Parkinson's disease (PD) and essential tremor (ET) are common movement disorders [46]. Deep brain stimulation (DBS), in which electrical leads are surgically implanted in the thalamic, subthalamic, or pallidal region of the brain, is a highly effective symptomatic treatment of these conditions [12]. The leads are connected to a subcutaneously implanted impulse generator (neurostimulator). Unlike ablative surgery, DBS is reversible and adaptable in the setting of progressively worsening disease. Over the past three decades, more than 100,000 patients have been treated with DBS around the world [39].

In many centers, DBS surgery is performed under local anesthesia to enable intraoperative stimulation tests [1] mostly through a specific exploration electrode, for direct observation of the therapeutic effect of stimulation and of side effects. The therapeutic effects induced by stimulation tests are visually evaluated and rated in different ways by different centers, but always with the same underlying concept: either the observer directly rates the improvement of a symptom (e.g., tremor) in response to stimulation, or the observer rates the severity of the symptom both without and with stimulation using a clinical scale such as the Unified Parkinson's Disease Rating Scale (UPDRS [9]). Previous studies have revealed that such ratings have a low inter-rater [26, 33] and intra-rater [33] reliability because of their subjectivity and their high dependence on the experience of the evaluating neurologist [11]. Moreover, pen and paper are used to note down the subjective ratings of the therapeutic effects and side effects that are observed at varying stimulation parameters and positions; retrospective comparisons once the testing is completed are difficult and dependent on human memory. If the measurement and evaluation of changes in tremor were performed quantitatively, these limitations could be overcome.

Numerous methods with different sensors, including EMG [2, 15, 17, 25, 44], spirograms [7, 37], and gyroscopes [23, 32, 38], have been used to quantify tremor. Accelerometers have been applied outside the operating room (OR) for a wide variety of purposes, e.g., to characterize pathological tremor $[8,13]$, to compare it with physiological tremor $[20,35,36]$, and to evaluate the severity and evolution of tremor [28, 29, 40] and the tremor-alleviating effect of drugs or DBS [22, 36, 47]. Pulliam et al. [34] used motion sensors during postoperative DBS pulse generator programming to develop automated programming algorithms and concluded that 
objective assessment can improve patients' outcomes. These methods, however, were developed to evaluate tremor outside the OR and cannot be used in their current form during surgery, for multiple reasons. The patient has only limited freedom of movement during surgery, compared to preoperative or postoperative examination; to be useful, intraoperative tremor assessment must be performed at many different positions of the test electrode and at a variety of stimulation current amplitudes; and the surgical team's access to the patient and the level of patient comfort are especially important considerations in the design of systems for intraoperative use.

For these reasons, unlike the numerous methods mentioned above for tremor assessment outside the OR, intraoperative quantitative tremor assessment has only rarely been described in the literature. These descriptions were mostly for research purposes, for example the one-time use of inertial sensors during a thalamotomy [21], the evaluation of the effect of non-constant interpulse intervals of DBS stimulation on tremor [4], or the identification of a target structure by spectral correlation of a tremor signal from goniometers with the electrophysiological signal from microelectrode recording [43]. To our knowledge, only Journee et al. [14] and Papapetropoulos et al. [27] evaluated tremor intraoperatively in a relatively large patient cohort. Their tests, however, seem to have been performed by stimulating through the chronic DBS lead in order to ascertain the optimal stimulation parameters, rather than through exploratory test electrodes of the type used in most centers to find the optimal target site for stimulation. None of these methods were used to help determine the best site for DBS lead implantation, nor were any of them implemented in more than a single surgical center or as a part of the regular surgical protocol for DBS. Additionally, to the best of our knowledge, the correlation between the intraoperative visual (subjective) and quantitative evaluations has not yet been thoroughly investigated.

This study presents a method designed for the specific purpose of quantitatively estimating changes in tremor during intraoperative stimulation tests through an exploratory electrode to identify the optimal position for implantation of the chronic lead in routine DBS surgery. It tries to overcome the limitations of previous methods by recording data in parallel to conventional subjective visual evaluation, by recording baseline activity before each stimulation test, and by synchronizing the data with the electrophysiology system. The aim of the present study was to evaluate the feasibility and adaptability of the method by applying it to 15 patients undergoing DBS surgery in two clinical centers. Furthermore, the correlations between the recorded 
accelerometric data and the visual evaluations during surgery were studied to better understand the similarities and differences of the two evaluation methods.

\section{Materials and Methods}

\section{Surgical protocol}

In order to design a method for intraoperative use, the DBS surgical procedure has to be understood, which, in most centers, can be summarized in 4 steps as follows: (1) the anatomical target and the best path to reach it are defined on the patient images during pre-surgical planning. An electrode trajectory or, in many cases, multiple closely spaced parallel trajectories through the target region are selected for intraoperative testing. (2) At surgery, intraoperative exploratory electrodes are inserted along the chosen trajectory or trajectories, and the target region is electrophysiologically mapped with microelectrode recording (MER). (3) After MER, stimulation test is administered at various locations, and the therapeutic effects and side effects are observed. The visually observed improvement in tremor (IV), the amplitude of the stimulating current that brought about this improvement (AV), and the lowest stimulation current amplitude at which a side effect is observed (side-effect threshold) are noted for each site of stimulation. (4) The site for implantation of the chronic DBS lead is chosen to be one with low AV and a large difference ("therapeutic window") between AV and the side-effect threshold. Optimally, the target site should be one among a group of adjacently located sites that all have a large therapeutic window.

We aimed to design the acceleration recording system to be usable in multiple clinical centers with few or no modifications. Patients from two different clinical centers were included: from the University Hospital in Clermont-Ferrand, France (Center 1), and from the Inselspital in Bern, Switzerland (Center 2). Although the basic surgical steps in these centers correspond to the ones described above, there are significant differences in how these steps are configured and executed. Table 1 lists the various surgical steps and the configuration used in the two centers which were considered when developing the quantitative symptom evaluation system. 


\begin{tabular}{|c|c|c|}
\hline Surgical step & Center 1 & Center 2 \\
\hline Pre-surgical planning & Direct visual targeting & $\begin{array}{l}\text { Combination of } \mathrm{AC} / \mathrm{PC} \text { based } \\
\text { and direct visual targeting }\end{array}$ \\
\hline $\begin{array}{l}\text { Number of trajectories per } \\
\text { hemisphere }\end{array}$ & 2 & 1 to 2 \\
\hline Intraoperative MER & Yes & Yes \\
\hline $\begin{array}{l}\text { No. of explored positions per } \\
\text { trajectory }\end{array}$ & $5-10$ & 15 or more \\
\hline $\begin{array}{l}\text { Distance of first position from } \\
\text { target }(=0 \mathrm{~mm})\end{array}$ & Based on pre-surgical planning & $10 \mathrm{~mm}$ \\
\hline Distance between positions & $1 \mathrm{~mm}$ & $\begin{array}{l}1 \mathrm{~mm}(5-10 \mathrm{~mm}) \\
0.5 \mathrm{~mm}(4.5 \mathrm{~mm} \text { to target })\end{array}$ \\
\hline $\begin{array}{l}\text { Intraoperative stimulation } \\
\text { tests through exploration } \\
\text { electrode }\end{array}$ & Yes & Yes \\
\hline Test positions & All MER positions & $\begin{array}{l}\text { Chosen based on MER data ( } 2 \text { to } \\
6 \text { per hemisphere) }\end{array}$ \\
\hline Stimulation pattern & Current-controlled & Current-controlled \\
\hline Range (mA) & $0-3$ & $0-4$ \\
\hline Step size $(\mathbf{m A})$ & 0.2 & 0.5 or 1 \\
\hline $\begin{array}{l}\text { Visual evaluation of baseline } \\
\text { tremor }\end{array}$ & $\begin{array}{l}\text { At every position just before start of test } \\
\text { stimulation }\end{array}$ & $\begin{array}{l}\text { Before starting test stimulation } \\
\text { of each hemisphere. }\end{array}$ \\
\hline $\begin{array}{l}\text { Documentation of findings of } \\
\text { intraoperative test stimulation }\end{array}$ & For each position of test stimulation & $\begin{array}{l}\text { For each position and amplitude } \\
\text { of test stimulation }\end{array}$ \\
\hline Level of improvement & $\begin{array}{l}\text { Maximum degree of improvement and the } \\
\text { stimulation amplitude that induced it }\end{array}$ & $\begin{array}{l}\text { The degree of improvement with } \\
\text { stimulation at that position and } \\
\text { amplitude }\end{array}$ \\
\hline Side effects & Type and amplitude & Type \\
\hline $\begin{array}{l}\text { Rating scale for tremor } \\
\text { evaluation }\end{array}$ & $\begin{array}{l}\text { Direct relative improvement rating; 0-4 } \\
\text { scale, worst (0) to best (4) }\end{array}$ & $\begin{array}{l}\text { Absolute rating based on } \\
\text { UPDRS; } 0-4 \text { scale, best (0) to } \\
\text { worst (4) }\end{array}$ \\
\hline Before stimulation & Baseline tremor defined as 0 & $\begin{array}{l}\text { Tremor severity based on } \\
\text { UPDRS scale }\end{array}$ \\
\hline Tremor arrest & 4 & 0 \\
\hline $\begin{array}{l}\text { Number of intermediate } \\
\text { levels (indicated using } \\
\text { underlining) }\end{array}$ & 1 level $(0, \underline{0.5}, 1, \underline{1.5} \ldots)$ & 2 levels $(4, \underline{4-}, \underline{3+}, 3, \underline{3-\ldots})$ \\
\hline $\begin{array}{l}\text { Choice of chronic implant } \\
\text { position for DBS lead }\end{array}$ & $\begin{array}{l}\text { Stimulation test position among a group of } \\
\text { adjacently located positions all having a } \\
\text { large therapeutic window }\end{array}$ & $\begin{array}{l}\text { Deepest stimulation test position } \\
\text { with a large therapeutic window }\end{array}$ \\
\hline Stimulating contact & \multirow{2}{*}{$\begin{array}{l}\text { Based on the adjacent positions having large } \\
\text { therapeutic windows }\end{array}$} & Distal contact (number 0) \\
\hline Contact border & & Distal border \\
\hline
\end{tabular}

Table 1 Details of the configuration and execution of surgical steps in the two clinical centers that were considered when designing the adaptable accelerometer recording system. 
In Center 1, stimulation tests are performed at various preoperatively chosen positions on the trajectories (between 10 and 18 per hemisphere) and only the highest improvement in tremor and the corresponding stimulation current amplitude are noted for each stimulation position (one improvement noted per position). In Center 2, stimulation tests are performed only at a few positions (between 2 and 6 per hemisphere) chosen on the basis of the electrophysiological activity observed during MER, but the improvement in tremor is noted for each stimulation current amplitude (between 4 and 8 improvements noted per position). In addition, in Center 1, the stimulation current is varied from 0 to $3 \mathrm{~mA}$ in steps of $0.2 \mathrm{~mA}$ for each stimulation test position, whereas, in Center 2, the stimulation current range goes up to $4 \mathrm{~mA}$ and the step size is decided based on the observed response of the patient. These differences significantly influence the data recording procedure.

The analysis of the acquired data is also altered because of some of the differences in the surgical procedure like the rating scales for tremor evaluation and the method used for choosing the chronic implant position of the DBS lead. In Center 1, the chronically stimulating lead is implanted at the position having a large therapeutic window itself as well as its adjacent positions, and the contact and its border are chosen in a manner permitting chronic stimulation at these adjacent positions if needed. In Center 2, the distal border of the distal contact is implanted at the deepest effective stimulation position making it possible to chronically stimulate other effective positions located proximally. Such differences should be considered when designing a method for intraoperative use in multiple clinical centers.

\section{Acceleration data recording}

A commercially available 3 -axis acceleration sensor evaluation board (STEVAL-MKI022V1, ${ }^{1}$ ST Micro, Geneva, Switzerland), with a sampling rate of $400 \mathrm{~Hz}$ and a range of $8 \mathrm{~g}$, was used to quantify changes in tremor. To facilitate its use in the OR, it was placed in an in-housedeveloped, non-conductive printed plastic case (FullCure 830 Vero White, Stratasys, Eden Prairie, USA) that can be attached to the patient's wrist with a Velcro strap (Fig. 1). The sensor evaluation board was interfaced with and powered by a laptop via a USB connection.

\footnotetext{
${ }^{1}$ STEVAL-MKI022V1 (data sheet: https://www.arrow.com/en/products/steval-mki022v1/stmicroelectronics or at authors) is no longer produced by the manufacturer. It has been replaced by STEVAL-MKI089V1 evaluation board (data sheet: http://www.st.com/content/st_com/en/products /evaluation-tools/product-evaluation-tools/mems-motion-sensor-eval-boards/steval-mki089v1.html) which uses the same accelerometer (LIS331DLH) as in the present study.
} 


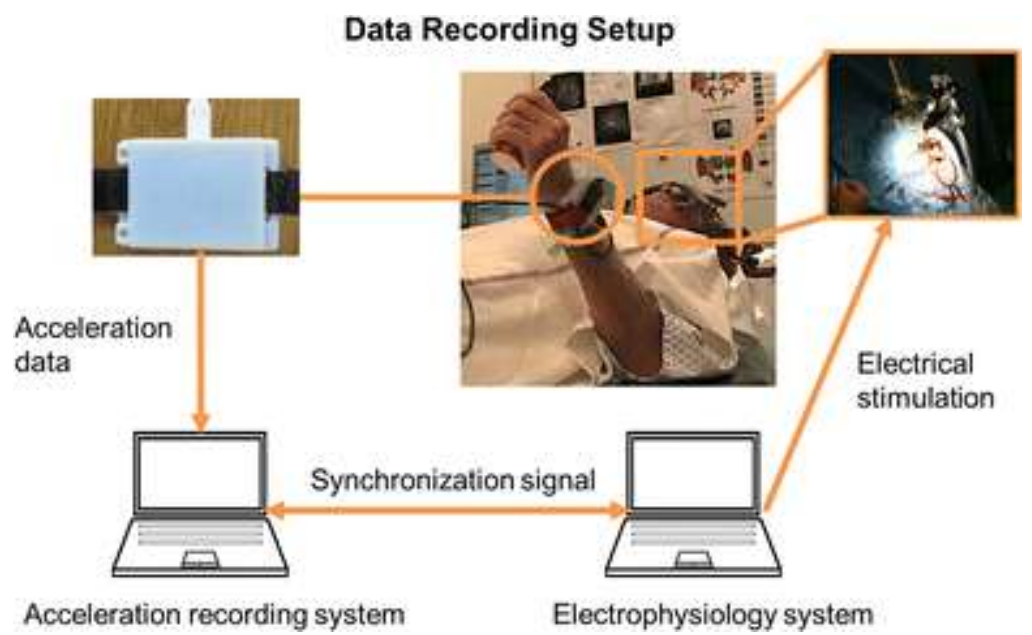

Figure 1. Intraoperative data recording setup. The acceleration sensor is inside a plastic case (top left), which is mounted on the patient's wrist with a Velcro strap. The sensor is connected to our recording system (bottom left), which is also connected to the DBS system (bottom right) so that data from the two sources can be synchronized.

The data recording setup was approved for use in clinical studies after multiple tests revealed its harmlessness to patients: The chosen 3D printing material was biocompatible. Heating tests performed by continuously recording acceleration data for $5 \mathrm{~h}$ marginally raised the temperature of the case by $2.5^{\circ} \mathrm{C}$; the maximum duration of continuous intraoperative recording was $15 \mathrm{~min}$, which would not lead to any degree of heating that would be appreciable by the patient. Nevertheless, the Velcro strap made it possible to remove the sensor at any time at the patient's request in case wearing it was uncomfortable. All the equipment was cleaned with disinfectant wipes before and after each use, and a new Velcro strap was used for each patient, to minimize potential sources of infection. To lessen the risk of leakage currents, the laptop was powered by battery only, rather than by line current.

For data recording and visualization, a computer application (LemurDBS) has been developed in our laboratory in Java (Oracle Corporation, California, USA). In order to make the system adaptable to the varying DBS procedures in different clinical centers, software profiles can be made for individual centers to customize LemurDBS during the initiation phase to adapt it to the center's surgical procedure. In addition, the software can be further adapted for individual operations by providing certain details of the operation, such as the number of trajectories or number of positions at which stimulation tests would be performed. The important information to be obtained intraoperatively, for example the position and amplitude of the stimulation test, any 
observed side effects, and the threshold amplitudes at which they arose, can be entered manually. During data recording, the acceleration data and extracted outcome measures (for details, see data analysis section) are visualized online to check the correct functioning of the system and to identify any fluctuation of the pre-stimulation baseline tremor. All data are stored for offline analysis.

For data synchronization, the acceleration data recording software was connected to the MicroGuide Pro (Alpha Omega Eng., Nazareth, Israel) or LeadPoint (Medtronic, Minneapolis, USA) electrophysiology systems that were used in the two centers for MER and stimulation tests (Fig. 1). A PhidgetInterfaceKit 2/2/2 board (Phidgets Inc. Calgary, Alberta, Canada) was used for this purpose. A 5 V CMOS signal was sent from LemurDBS to the MicroGuide Pro system at the beginning and end of each acceleration data recording. For the LeadPoint system, synchronization was obtained by acquiring time-stamped analog stimulation signal as measured by the non-stimulating electrode $2 \mathrm{~mm}$ away from the stimulating one.

As MER recording systems are very sensitive to noise, wired connections were used between the laptop, the acceleration sensor, and the electrophysiology system. The wireless system on the laptop was disabled to ensure that no wireless signals were emitted from our recording setup that could interfere with other systems in the OR.

No specific instructions were given to the patient or the surgical team for the data recording, which did not require any conscious effort or participation on their part and therefore did not prolong the operations.

\section{Clinical application}

Quantitative evaluation of change in tremor was carried out during DBS implantations of 15 patients, 9 in Center 1 and 6 in Center 2. All patients were good candidates for DBS according to the international guidelines [16]. They gave written informed consent before surgery, and the experimental procedures were approved by the respective Institutional Ethics Committee (Center 1: 2011-A00774-37/AU905; Center 2: 2365-multicenter study together with the University Hospital in Basel). The details of surgery for each patient, including the number of trajectories explored and the number of stimulation tests on each trajectory, are provided in Table 2. 


\begin{tabular}{|c|c|c|c|c|c|}
\hline \multirow[t]{2}{*}{ Patient } & \multirow[t]{2}{*}{$\begin{array}{l}\text { Surgical } \\
\text { Center }\end{array}$} & \multirow[t]{2}{*}{ Disease } & \multirow[t]{2}{*}{$\begin{array}{l}\text { Target } \\
\text { structure }\end{array}$} & \multicolumn{2}{|c|}{$\begin{array}{l}\text { Trajectory position (number of stimulation test positions } \\
\text { on this trajectory) }\end{array}$} \\
\hline & & & & Left side & Right side \\
\hline \multirow[t]{2}{*}{1} & \multirow[t]{2}{*}{ Center 1} & \multirow[t]{2}{*}{ PD } & \multirow[t]{2}{*}{ STN } & Central (6) & Central (9) \\
\hline & & & & Posterolateral (5) & Posterior (9) \\
\hline \multirow[t]{2}{*}{2} & \multirow[t]{2}{*}{ Center 1} & \multirow[t]{2}{*}{ ET } & \multirow[t]{2}{*}{ VIM } & Central (8) & Central (7) \\
\hline & & & & Posterolateral (8) & Posterolateral (8) \\
\hline \multirow[t]{2}{*}{3} & \multirow[t]{2}{*}{ Center 1} & \multirow[t]{2}{*}{ ET } & \multirow[t]{2}{*}{ VIM } & Central (6) & Central (7) \\
\hline & & & & Posterior (6) & Posterior (6) \\
\hline \multirow[t]{2}{*}{4} & \multirow[t]{2}{*}{ Center 1} & \multirow[t]{2}{*}{ ET } & \multirow[t]{2}{*}{ VIM } & Central (5) & Central (7) \\
\hline & & & & Posterior (5) & Posterior (7) \\
\hline \multirow[t]{2}{*}{5} & \multirow[t]{2}{*}{ Center 1} & \multirow[t]{2}{*}{ ET } & \multirow[t]{2}{*}{ VIM } & Central (8) & Central (8) \\
\hline & & & & Posterior (8) & Posterior (8) \\
\hline \multirow[t]{2}{*}{6} & Center 1 & ET & VIM & Central (9) & Central (5) \\
\hline & & & & Posterior (9) & Posterior (5) \\
\hline \multirow[t]{2}{*}{7} & Center 1 & PD & VIM & Central (7) & Central (7) \\
\hline & & & & Posterior (7) & Posterior (7) \\
\hline \multirow[t]{2}{*}{8} & Center 1 & PD & STN & Central (7) & Central (6) \\
\hline & & & & Posterolateral (7) & Posterolateral (6) \\
\hline \multirow[t]{2}{*}{9} & Center 1 & ET & VIM & Central (8) & Central (8) \\
\hline & & & & Posterior (8) & Posterior (8) \\
\hline \multirow[t]{2}{*}{10} & Center 2 & PD & STN & Central (2) & Central (1) \\
\hline & & & & & Lateral (1) \\
\hline 11 & Center 2 & PD & STN & Central (2) & Central (2) \\
\hline & & & & & Medial (2) \\
\hline 12 & Center 2 & PD & STN & Central (2) & Central (2) \\
\hline & & & & Lateral (1) & Lateral (1) \\
\hline 13 & Center 2 & PD & STN & Central (2) & Central (4) \\
\hline & & & & Medial (2) & Medial (4) \\
\hline & & & & Posterior (1) & Posterior (2) \\
\hline 14 & Center 2 & ET & VIM & Central (2) & Central (2) \\
\hline & & & & Medial (2) & Medial (2) \\
\hline 15 & Center 2 & PD & STN & Central (3) & Central (2) \\
\hline
\end{tabular}

Table 2 Details of the included patients and their surgical procedures STN subthalamic nucleus, VIM ventral intermediate nucleus of the thalamus.

In Center 1, for patient 1, rigidity was also evaluated by the neurologist for short periods of $2-5 \mathrm{~s}$ and subsequently recorded during stimulation tests by moving the patient's forearm. However, rigidity was only evaluated at stimulation amplitudes at which the tremor was suppressed by stimulation. Patient 7 also exhibited rigidity as a symptom. However, during surgery, only the tremor was evaluated. Because of a software error during the implantation of the left hemisphere of patient 7 , no synchronization signal was sent to the electrophysiology system. Hence, data 
from the left hemisphere could not be analyzed. The problem had no influence on the operation itself and was resolved before the neurosurgeons proceeded to the right hemisphere.

In Center 2, for patient 10, during the stimulation of the right hemisphere, no tremor was observed and rigidity was evaluated by a neurologist during the surgery. Patient 12 had more tremor in the left lower limb than in the left hand; therefore, to test the versatility of the method, the acceleration sensor was mounted on the foot in the distal metatarsal region. The visual evaluation was also based on rest tremor reduction in the patient's foot. During implantation in the right hemisphere of patient 11, the acceleration sensor was unintentionally disconnected from the recording software, and for the right hemisphere of patient 13, no acceleration data were recorded because of waning battery power in the recording laptop.

\section{Data analysis}

The raw data recorded during DBS surgery as well as a first analysis were visualized in real time in LemurDBS during surgery. For ethical reasons, the results of the acceleration data analysis were not considered when the chronic implant position for the DBS lead was chosen (the study had been declared a purely observational study of the potential usefulness of a new method, and any influence of the intraoperative accelerometric findings on surgical decision making was explicitly ruled out). An exhaustive data analysis was performed postoperatively in MATLAB (Mathwork Inc., Massachusetts, USA) including a comparison between results of the accelerometric and visual evaluations. Statistical analysis was performed with SOFA Statistics (Paton-Simpson \& Associates Ltd, Auckland, New Zealand) and OriginPro (OriginLab Corporation, Massachusetts, USA).

\subsection{Preprocessing}

As a first step, the magnitude (square root of sum of squares) of every sample of the 3 different axes of acceleration data was calculated. In general, acceleration signals corresponding to movements other than tremor were also present in the recorded data and could be clearly identified visually. Large movements, like those corresponding to rigidity evaluations, were ignored for real-time analysis and were manually eliminated from the data sets during postoperative evaluation [48]. It was necessary to filter the acceleration data to extract the tremor signal while suppressing the effect of gravity and higher-order spectral harmonics. In addition, 
the filters had to be optimized for low computation time to allow for real-time evaluation. While previous studies have shown that PD and ET have a dominant frequency between 3 and $12 \mathrm{~Hz}$ [18], our data showed a range of 3-6 Hz. Based on this, a 2-step process was employed to filter the data. (1) A time-varying high-pass filter called "smoothness priors" [48], with a cutoff frequency of $2 \mathrm{~Hz}$ [20], was used to remove low-frequency trends and the effect of gravity. (2) A second-order Butterworth low-pass filter was tested with cutoff frequencies from 10 to $30 \mathrm{~Hz}$ in steps of $5 \mathrm{~Hz}$. In the present study, a cutoff frequency of $10 \mathrm{~Hz}$ was used because of adequate suppression of higher-order (2 and more) harmonics and digital noise without altering the outcome measures or the calculated improvement in tremor. Nevertheless, the cutoff frequency can be adapted to accommodate unexpected variations in tremor frequency. For postoperative analysis, synchronization markers and stimulation amplitude from the data for each stimulation test position were imported into MATLAB. For data coming from the Alpha Omega system, Neuroexplorer (Nex Technologies, Madison, Alabama, USA) was used to read the proprietary format. Acceleration data were imported and synchronized with the stimulation amplitude (Fig. 2, top). Correct synchronization was verified by visual inspection.

\subsection{Outcome measures}

To estimate the changes in tremor during intraoperative stimulation tests, the accelerometer data were analyzed in a windowed manner. Various factors such as the average duration per stimulation amplitude, the sampling rate, and the range of tremor frequency had to be taken into account in choosing the window length. Based on these factors, windows of 1-4 s of time length and 0-50 \% overlap were tested, and a non-overlapping window of $2 \mathrm{~s}$ was found to be optimal for data analysis. For each measurement position, outcome measures (standard deviation (1),

signal energy (2), entropy (3), dominant frequency (4), and spectral amplitude of the dominant frequency (5)) were extracted from data recorded during baseline and stimulation periods:

$$
\begin{aligned}
& \text { Standard deviation }=\sqrt{\frac{1}{N-1} \sum_{n=1}^{N}\left|x_{n}-\mu\right|^{2}} \\
& \text { with } \mu=\frac{1}{N} \sum_{n=1}^{N} x_{n} \\
& \text { and } N=\text { Total number of samples }
\end{aligned}
$$



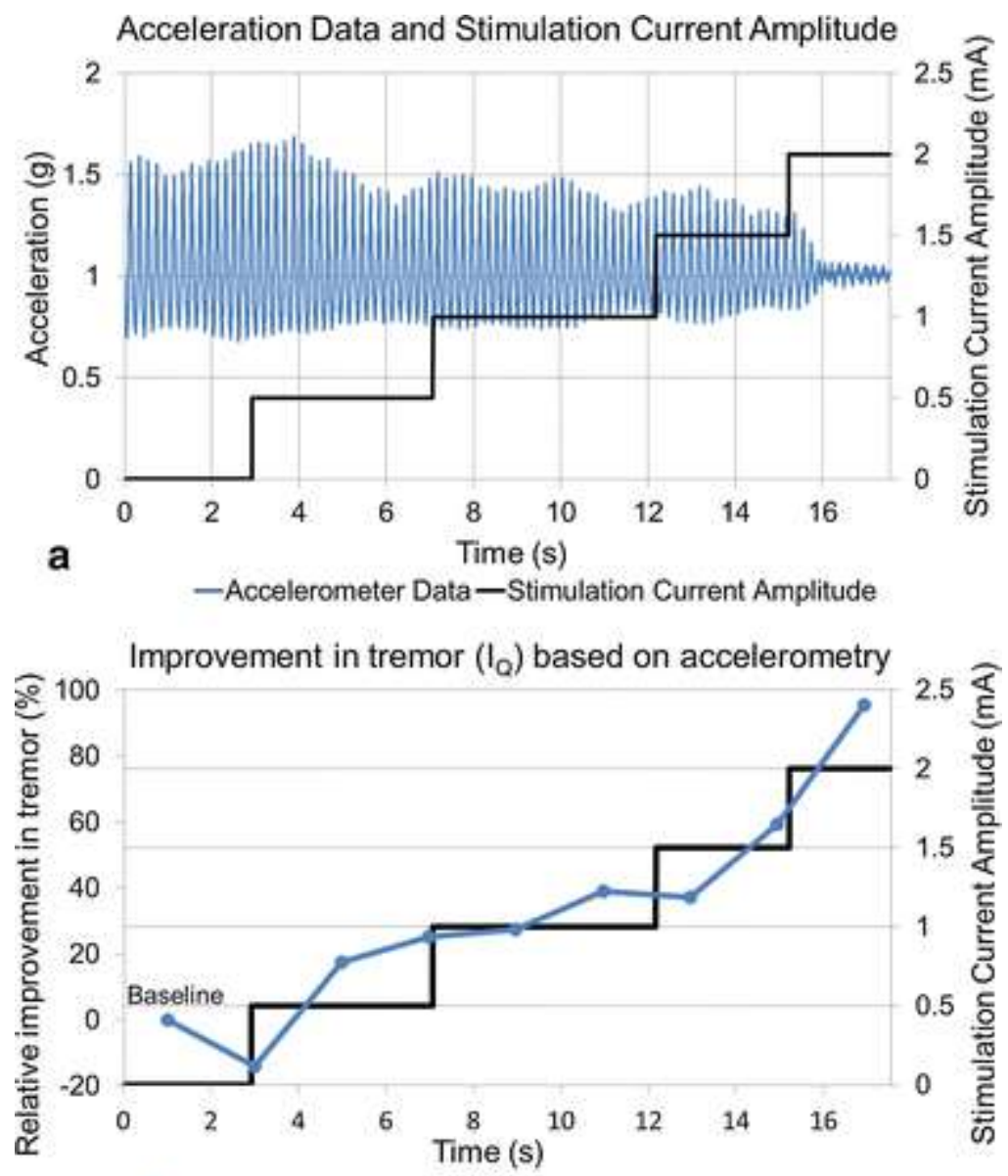

b - Improvement in tremor based on accelerometric evaluation -Stimulation Current Amplitude

Figure 2. a Raw acceleration data (blue signal) recorded in synchronization with the stimulation amplitude (black stepped line). $\boldsymbol{b}$ Improvement in tremor estimated from the outcome measures extracted from accelerometer data (IQ) for the different stimulation current amplitudes (color figure online). 
Signal energy $=\sum_{n=1}^{N}\left|x_{i}\right|^{2}$
Entropy $=-\sum_{n=1}^{N} p_{n} \log _{2} p_{n}$ where $p$ is

the probability density function

Dominant frequency $=k$ such that $\max \left(X_{k}\right)$ where

$$
\begin{aligned}
& X_{k}=\sum_{n=0}^{N-1} x_{n} e^{-i 2 \pi k \frac{n}{N}} \\
& \text { and } k=0, \ldots, N-1
\end{aligned}
$$

Spectral amplitude of dominant frequency $=\max \left(X_{k}\right)$

$$
\text { where } X_{k}=\sum_{n=0}^{N-1} x_{n} e^{-i 2 \pi k \frac{n}{N}} \text { and } k=0, \ldots, N-1
$$

However, statistical tests showed that 3 outcome measures (standard deviation, signal energy, and spectral amplitude of the dominant frequency) were more sensitive toward changes in tremor [41], and only they were retained for further analysis. The extracted measures were graphically presented along with the stimulation current amplitude for visual analysis. Once the stimulation test was completed, the time window representing the highest tremor in the baseline data was identified and selected. The measures (a set of all three) extracted from this baseline window were used to normalize (6) the respective measures extracted for the following windows obtained during the stimulation test.

$$
\begin{aligned}
& \text { Normalized outcome measure } \\
& \qquad=\left(\frac{\text { Baseline value }- \text { current value }}{\text { Baseline value }}\right) \times 100 \%
\end{aligned}
$$

Such normalization permitted a relative evaluation of tremor with changing stimulation current amplitude. The mean (7) of the normalized standard deviation, signal energy, and spectral amplitude of dominant frequency for any given window was termed as quantitatively calculated improvement in tremor or IQ (Fig. 2, bottom). 


$$
\begin{aligned}
I_{\mathrm{Q}}= & \frac{1}{3} \times\left(\begin{array}{c}
\text { Normalized } \\
\text { standard deviation }
\end{array}+\begin{array}{c}
\text { Normalized } \\
\text { signal energy }
\end{array}\right. \\
& \left.+\begin{array}{c}
\text { Normalized spectral } \\
\text { amplitude of dominant frequency }
\end{array}\right)
\end{aligned}
$$

\subsection{Comparative postoperative analysis}

To establish the benefits of accelerometric tremor evaluation during DBS over visual evaluation, the first step was to compare the improvement in tremor identified by the two methods. To compare the discrete levels of the rating scales used for visual evaluations to the continuous values of accelerometric evaluation and to eliminate the difference between the rating scales used by the two different centers, the improvement values were classified in 5 categories as described

\begin{tabular}{|c|c|c|c|c|c|c|}
\hline \multirow[t]{3}{*}{ Category } & \multirow[t]{3}{*}{ Descriptive evaluation in tremor } & \multirow{3}{*}{$\begin{array}{l}\text { Quantitative } \\
\text { accelerometry- } \\
\text { based evaluation } \\
\text { (IQ) }(\%)\end{array}$} & \multicolumn{4}{|c|}{ Visual evaluation (IV)a } \\
\hline & & & \multirow{2}{*}{$\begin{array}{c}\text { Center 1: } \\
\text { Direct } \\
\text { rating }\end{array}$} & \multicolumn{3}{|c|}{$\begin{array}{l}\text { Center 2: Rating using absolute } \\
\text { UPDRS }\end{array}$} \\
\hline & & & & Baseline $=$ & aseline $=$ & aseline $=2$ \\
\hline A & Tremor arrest & $>87.5$ & 4 & 0 & 0 & 0 \\
\hline B & High improvement & $75 \pm 12.5$ & $3,3.5$ & $\begin{array}{l}1+, 1,1- \\
0+\end{array}$ & $1-, 0+$ & $0+$ \\
\hline $\mathrm{C}$ & Average improvement & $50 \pm 12.5$ & $2,2.5$ & $2+, 2,2-$ & $2-, 1+, 1$ & $1+, 1,1-$ \\
\hline $\mathrm{D}$ & Limited improvement & $25 \pm 12.5$ & $\begin{array}{l}0.5,1 \\
1.5\end{array}$ & $\begin{array}{l}4-, 3+, 3 \\
3-\end{array}$ & $3-, 2+, 2$ & $2-$ \\
\hline $\mathrm{E}$ & $\begin{array}{l}\text { No improvement/tremor } \\
\text { worsening }\end{array}$ & $<12.5$ & 0 & 4 & 3 & \\
\hline
\end{tabular}
in Table 3.

Table 3 Categories used for classification of tremor improvement for the different rating scales used for visual-and accelerometer-based evaluations. aDetails about the two different clinical scales are given in Table 1. 
Since in Center 1 the relative improvement in tremor was directly visually rated, the categories were easy to assign. In Center 2, tremor severity was rated on the UPDRS scale, i.e., in absolute rather than relative terms (see Table 1 for details). In consequence, the baseline severity had to be considered to determine the corresponding improvement values. Table 3 shows the baselinedependent classification. As no patient had a baseline rating of 1, it is not listed in the table.

The classification of improvement values from visual evaluation (IV) was straightforward, as only one IV value was available for each stimulation current amplitude. Because the quantitative improvement values (IQ) were calculated in a windowed fashion, multiple values were available depending on the number of windows that were completely enclosed in the period of a given stimulation current amplitude. Therefore, for the classification as well as for the comparison with IV at any given stimulation current amplitude, the IQ values for the same stimulation current amplitude were averaged. To study the distribution (pairwise) of IV and IQ values, the Wilcoxon two-sided signed rank test [50] was used to compare their population mean ranks. Also, as IV and IQ are both tremor improvement values, a positive linear correlation should exist between these two data sets. To check statistically for such a correlation, Spearman's test [45] was used.

In addition to comparing the improvement in tremor identified by the different methods, the IQ values were also used to identify effective stimulation current amplitudes. For every stimulation test position, the lowest stimulation current amplitude (mA) at which the IQ value was similar to the IV value (highest IV value for Center 2) was identified and termed as the quantitatively identified effective stimulation current amplitude (AQ). The Wilcoxon two-sided signed rank test was used to compare AQ values to the visually identified effective amplitude (AV) values. To study the effect of using accelerometric evaluation of tremor on the implant position of the permanent DBS lead, the clinical staff was given the AQ values after implantation and asked to state where they would have implanted the permanent DBS lead on the basis of these values rather than AV values. 


\section{Results}

The presented setup and method were successfully applied to the intraoperative stimulation tests in both the clinical centers. The data recording setup had certain failures during the surgery of 4 patients. While the synchronization failure for patient 6 was due to a software error, the loss of battery power for patient 13 was due to human error. The disconnection between the sensor and the software during the surgery for patient 11 was rectified after the surgery with the use of a cable loop on the plastic case of the sensor board. Also, the synchronization with the LeadPoint system highlighted problems of signal saturation. On the other hand, the data analysis techniques were successful in eliminating noise and in extracting relevant information from the raw acceleration data.

In total, from all 15 patients, accelerometry data for 359 stimulation current amplitudes (223 in Center 1; 136 in Center 2) and the respective visually observed improvement values in tremor were acquired and analyzed offline. The Wilcoxon two-sided signed rank test $(p=0.041)$ showed that for any given improvement in tremor, the IV and IQ values are not significantly different. The result of the Spearman's test confirmed that for increasing improvement in tremor, IQ and IV values increase in a correlated manner $(\mathrm{R}=0.661, \mathrm{p}<0.001)$.

Figure 3a shows the counts of the quantitatively evaluated improvement IQ as a function of the corresponding visually assessed improvement IV in terms of categories as defined in Table 3. For example, if for one stimulation current amplitude, the change in tremor was visually assessed as average improvement (category $\mathrm{C}$ ) and quantitatively as $70 \%$ improvement (category $\mathrm{B}$ ), then this evaluation would fall in the group CB (column 3, row 2) in Fig. 3. Ideally, all the evaluations would fall in one of the groups on the $45^{\circ}$ diagonal, implying that both methods identify similar improvement in tremor for all the ranges. In fact, only 156 (43.5\%) evaluation pairs fell in the same category for both evaluation methods (Fig. 3b). Of the remaining 203 evaluations, IQ values were lower than IV values for 93 (26\%) (Fig. 3b, groups below the diagonal) and IQ values were higher for 110 (30.5\%) (Fig. 3b, groups above the diagonal). Further, 296 (82.5\%) of the evaluations fell in the same or adjacent categories (neighborhood, Fig. 3c), while the remaining $63(17.5 \%)$ evaluations showed differences of at least 2 categories between the two values (outliers, Fig. 3d). As mentioned in Table 1, in Center 1, only the maximum improvement in tremor was noted for every stimulation test. This, along with the higher number of patients 
from Center 1, creates a bias in the results as evident from the number of evaluations in category A for both IV and IQ values (Fig. 3a).

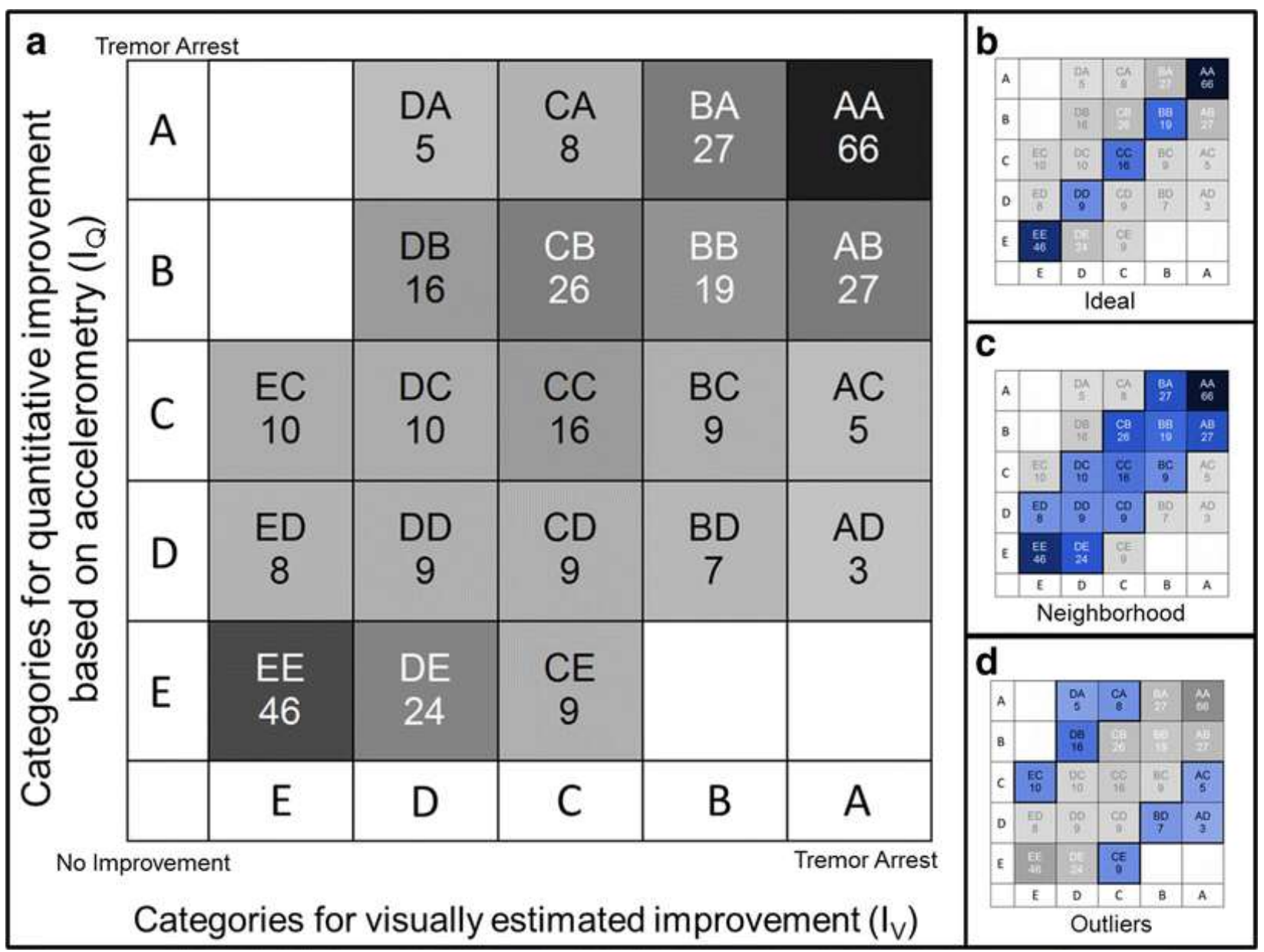

Figure 3. a $5 \times 5$ Heatmap illustrating the number of evaluations falling in each category pair, based on Table 3. The intensity of gray is proportional to the number of evaluations. (Right) Subdivision of the heatmap of the left into 3 scenarios: $\boldsymbol{b}$ the ideal scenario would be that all the evaluations fall along the diagonal meaning that visual and quantitative evaluation are equal; $c$ inclusion of the neighborhood around the diagonal, i.e., taking account as well variations of one category between the two evaluation methods; $\boldsymbol{d}$ considering the outliers where the difference between the visual improvement and the quantitative improvement is of at least two categories (color figure online).

The comparison between effective stimulation current amplitudes AV and AQ is depicted in Fig. 4. $\mathrm{AQ}$ values (mean $\pm \mathrm{SD}: 1.1 \pm 0.8 \mathrm{~mA})$ were significantly lower $(\mathrm{p}<0.001)$ than $\mathrm{AV}$ values $(1.7 \pm 0.8 \mathrm{~mA})$. The consideration of the acceleration data instead of the visual evaluations would have affected the choice of the chronic implant position for the DBS lead. Out of the 26 (Center 1: 18, Center 2: 8) choices, 15 (Center 1: 15, Center 2: 0) would have been different, and for 2 
implantations (Center 1: 2, Center 2: 0), a position on a different trajectory would have been chosen.

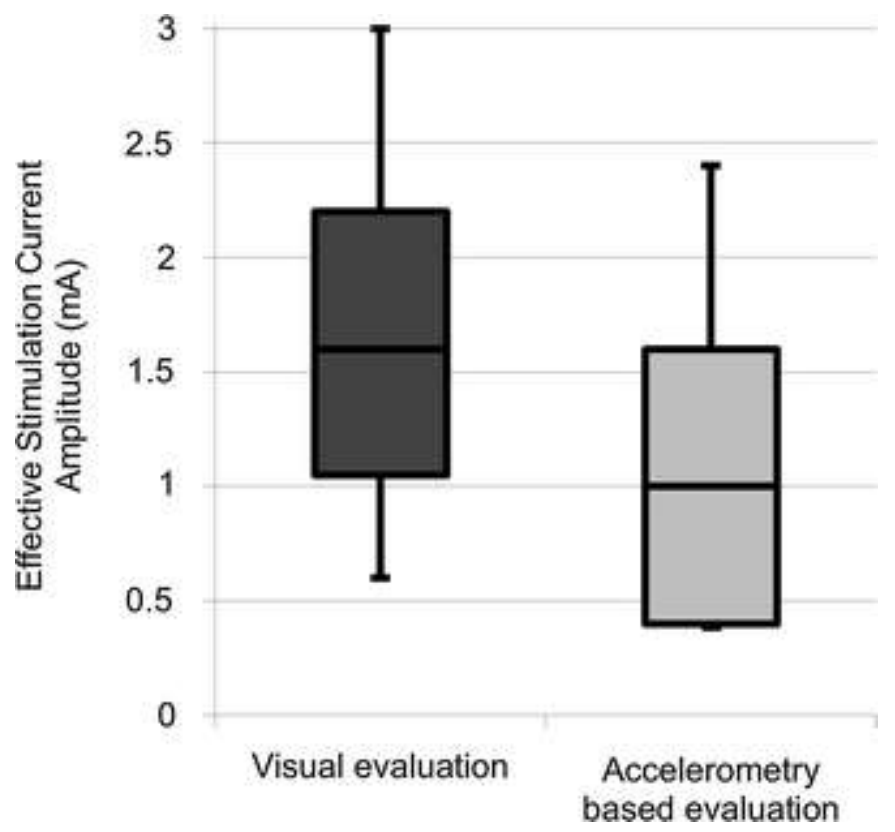

Figure 4. Box plot comparing the effective stimulation current amplitudes identified from visual evaluation $(A V)$ and quantitative evaluation $(A Q)$. The lower whiskers indicate 5 th and the upper whiskers 95th percentile of the values.

\section{Discussion}

The aim of this study was to provide an assistive tool supporting the neurologists in their tremor assessment during DBS surgery normally performed by visual inspection (the method now used in most centers). With the versatile system based on accelerometry, improvement in tremor can be measured quantitatively and any evaluation performed during DBS surgery can be revisited and visualized. The system was specifically designed to be used in the operating room during stimulation tests through exploration electrodes in different clinical centers, without impeding or prolonging the surgical procedure.

Some researchers have performed intraoperative quantitative evaluations to identify the best stimulation parameters for stimulating through the chronically implanted DBS lead. Journee et al. [14] performed intraoperative neurophysiological measurements with multiple sensors, including two uniaxial accelerometers, on a large patient cohort. They evaluated tremor by comparing it with a common baseline recorded before any incision. This method of comparison ignores the 
improvement in tremor that is often observed after insertion of the electrode but before any stimulating current is turned on (the so-called micro-lesional effect [24]). A baseline recording performed before each stimulation test, as in the current method, is necessary to have an accurate objective evaluation of improvement in tremor. Papapetropoulos et al. [27] used the commercially available CATSYS system (Danish Product Development Ltd., Snekkersten, Denmark) to evaluate the best stimulation parameters for PD patients undergoing DBS immediately after the DBS lead was placed in the brain. However, their method required the active participation of the patient by holding the tremor pen in a certain position, and the patients were given practice time to familiarize themselves with the testing procedure. Varying levels of familiarity with the system increase the subjectivity of such evaluations. In the method presented here, the acceleration data were recorded passively and in parallel to the routine visual evaluation.

Quantitative evaluation of improvement in tremor with accelerometers depends heavily on the filtering parameters used and the outcome measures extracted from the data. Gravity also has an effect on the data measured by the accelerometer [30] and therefore has to be corrected for before any outcome measures are extracted. In contrast to previously proposed methods, in which the effect of gravity was not suppressed, our method uses a cutoff frequency of $2 \mathrm{~Hz}$ for the smoothness-priors detrending method which has been shown to suppress the effect of gravitational acceleration on the raw data [20]. With regard to the outcome measures, as Papapetropoulos et al. [27] used a commercial system; they were restricted to the outcome measures available from it, i.e., tremor intensity, center frequency, its standard deviation, and harmonic index. Journee et al. [14] relied on spectral outcome measures without any filtering of the data. Additionally, they extracted temporal outcome measures from displacement estimated from the accelerometer data after double integration, which also significantly amplifies the noise in the accelerometer data [49]. In contrast, the linear outcome measures (temporal and spectral) like the ones proposed in this study have been shown to correlate with the UPDRS tremor scores during routine non-surgical clinical evaluation [20].

The use of our method in 15 patients in 2 different centers has already revealed some of its benefits and limitations. The complete setup was initially designed for use with the MicroGuide Pro system and later adapted for use with the LeadPoint system. Imperfect adaptation might 
underlie the signal saturation that was initially observed during synchronization with LeadPoint system but subsequently eliminated after the present study was conducted by modification of certain parameters in LemurDBS. This experience only underscores the need to test any quantitative symptom evaluation method in multiple clinical centers. A clear advantage of the method is the absence of any patient discomfort. The acceleration sensor is easy to attach to the patient's affected (usually upper) limb and to remove from it afterward; aside from patient comfort, this also ensures that the device does not block the surgical team's access to any part of the patient's body if needed (for insertion of new intravenous lines, etc.).

The comparison of improvement in tremor identified visually (IV) with that calculated from the acceleration data (IQ) is shown in Fig. 3. It must be noted, however, that a categorization of evaluations based on Table 3 results in loss of information, partly because of the large ranges of quantitative improvement values for each category and also because any worsening in tremor is also categorized in E, i.e., "No change/tremor worsening." Despite this loss of information, the number of evaluations in each category provides a better understanding of the similarities and differences between the two tremor evaluation methods. The results show that for $43.5 \%$ of the evaluations, both the methods identified similar improvement in tremor, i.e., IV and IQ values fell in the same category (Fig. 3b). In practice, however, minor changes in tremor are difficult to estimate visually, especially when the baseline tremor is small. Therefore, it is plausible that IQ values would be in the same category as the IV values, or at least in a neighboring category. In this scenario, $82.5 \%$ of the evaluations are either in the same or neighboring categories (Fig. 3c). The remaining $17.5 \%$ of evaluations show very large differences between the visual evaluation and the accelerometric evaluation (Fig. 3d).

One of the primary objectives of intraoperative stimulation tests is to identify sites where stimulation suppresses tremor. The high number of evaluations in the AA group would indicate that both methods can be used for this purpose. However, considering that the total number of the evaluations where only one of the methods indicated tremor arrest $(\mathrm{AB}, \mathrm{AC}, \mathrm{AD}, \mathrm{BA}, \mathrm{CA}$, and DA) is 76, it seems that a small residual tremor might not always be visually identified. As both methods estimate change in tremor compared to a baseline condition, the difference in estimation by the two methods may be a result of different choices of baseline. The visual evaluation is based on the complete baseline activity before test stimulation, whereas the accelerometric 
evaluation is based on the worst tremor ( $2 \mathrm{~s}$ long) in the whole baseline recording. Further, it may be possible that in case of very low baseline tremor, it was considered as suppressed by visual estimation, while the accelerometric evaluation only measured $50 \%$ improvement. Another possible reason for such differences might be that the evaluator did not retain an accurate memory of the observed baseline tremor while performing the evaluation. This emphasizes the need of an evaluation system that lets the evaluator re-check the severity of tremor at any time during the surgery. Previous studies proposing quantitative evaluation methods have shown similar findings $[14,27,36]$ suggesting that the limitations of current visual evaluation methods could be overcome by supplementing them with quantitative methods.

The impact of quantitative tremor evaluation on the DBS surgery can be gauged by its influence on surgical decision making, i.e., the choice of the site where the chronic DBS lead is finally implanted. One of the factors that influence this choice is the therapeutic window, i.e., the difference between the amplitude of stimulating current that results in an appreciable clinical effect and the side-effect threshold. As evident from Fig. 4, the quantitatively identified amplitude for effective stimulation (AQ) tends to be lower than the corresponding value obtained by visual evaluation (AV) and is thus associated with a wider therapeutic window. The evidence of this expansion in range affecting the choice of chronic implant position is provided by the results of comparison between clinical choices and choices based on quantitative data for the chronic implant position. The results show a stark difference in the choices between Center 1 and Center 2 because of the differences in the method of choosing the chronic implant position (Table 1). In Center 1, the stimulation test position among a group of adjacently located positions with large therapeutic window is chosen as opposed to Center 2, where the deepest effective stimulation test position is chosen. Also, as the number of stimulation tests per hemisphere is lower in Center 2, the choice of chronic implant position of the DBS lead would be less influenced by the accelerometer-based improvement values. A clinical study would be needed to determine the impact of different methods of choosing the chronic implant site on the ultimate clinical efficacy of stimulation.

We infer from our data analysis that the recording of a sufficient amount of baseline data is important. In the case of insufficient baseline data $(<5 \mathrm{~s})$ at a position, the analysis has to be done with the baseline from the previous position. However, this scenario does not significantly limit 
the method. Additionally, as the data analysis is performed with windows of $2 \mathrm{~s}$, each stimulation tests at any particular amplitude should be longer than that to increase the reliability of the result. A shorter duration might result in an incorrect identification of the effective amplitude of the stimulating current. Such errors will be smaller if the current is raised in smaller increments.

The results of this study show that our quantitative tremor evaluation method can help improve the placement of the chronic DBS lead. As a next step, a visualization tool will also be added to the software to allow the surgical team to see the results superimposed on the patients' brain scans (MRI or CT). To allow automatic identification of effective stimulation current amplitudes, thresholds for the quantitatively calculated improvement in tremor will be identified. For patients with Parkinson's disease, the quantification of tremor alone may not be sufficient. Rigidity is also present in these patients and is clinically evaluated during DBS surgery. Moreover, rigidity seems to be less affected by other factors like psychological stress, pain, alertness, microlesioning effects. Thus, in patients with Parkinson's disease a quantitative evaluation of rigidity is also needed for a comprehensive quantification of stimulation test results. Rigidity can be measured with intraoperative accelerometry as well [42].

Recent years have seen the development and marketing of new types of DBS leads. The idea of directional stimulation $[5,19]$ has been extensively researched, and new leads $[6,31]$ are already undergoing clinical trials. With the aid of quantitative methods as proposed in the current study, a more robust comparison can be made between different stimulation parameters and positions, and the time needed for testing may be shortened. Another area of increasing research is closed-loop DBS. Closed-loop systems have been proposed that are based not only on electrophysiological signals [10], but also on EMG and acceleration signals of tremor [3]. For such technologies to be practically useful and rapidly applicable, intraoperative quantitative evaluations of disease manifestations such as tremor and rigidity might play an important role. 


\section{Conclusion}

In this paper, we describe a new method in which an acceleration sensor is used for the quantitative evaluation of improvement in tremor in patients undergoing DBS for movement disorders. The method can be used in different surgical centers with little or no change of the system setup. It improves upon the previously proposed methods by using better filtering techniques and outcome measures that correlate with tremor severity. Accelerometry-based tremor evaluation widens the apparent therapeutic window of stimulation for tremor; it can therefore alter the exploratory test stimulation results and thus affect the choice of site for chronic DBS lead implantation. In the present study, the site of chronic lead implantation would have been different in $60 \%$ of cases if the surgeons had been allowed to consider the accelerometric evaluations instead of the subjective visual evaluations of tremor. Our preliminary results suggest that the limitations of the current clinical rating methods can be overcome by supplementing them with objective evaluation methods and, in turn, improve the determination of the optimum site for lead placement. To confirm the present findings, the method will have to be used in more patients undergoing DBS surgery.

\section{Acknowledgments}

This research was funded by the Swiss National Science Foundation and partly by the Germaine de Staël program of the Swiss Academy of Engineering Sciences. The authors acknowledge the contribution of Ms. Katrin Petermann in the programming of the connection of the LeadPoint system for synchronization with the acceleration data recording system. 


\section{References}

1. Abosch A, Timmermann L, Bartley S, Rietkerk HG, Whiting D, Connolly PJ et al (2013) An international survey of deep brain stimulation procedural steps. Stereotact Funct Neurosurg 91(1):1-11. doi:10.1159/000343207

2. Askari S, Zhang M, Won DS (2010) An EMG-based system for continuous monitoring of clinical efficacy of Parkinson's disease treatments. In: Conference proceedings: Annual international conference of the IEEE engineering in medicine and biology society. IEEE engineering in medicine and biology society. Conference, 2010, pp 98-101. doi:10.1109/IEMBS.2010.5626133

3. Basu I, Graupe D, Tuninetti D, Shukla P, Slavin KV, Metman LV et al (2013) Pathological tremor prediction using surface electromyogram and acceleration: potential use in 'ON-OFF' demand driven deep brain stimulator design. J Neural Eng 10(3):36019. doi:10.1088/1741-2560/10/3/036019

4. Birdno MJ, Kuncel AM, Dorval AD, Turner DA, Grill WM (2008) Tremor varies as a function of the temporal regularity of deep brain stimulation. NeuroReport 19(5):599-602

5. Chaturvedi A, Foutz TJ, McIntyre CC (2012) Current steering to activate targeted neural pathways during deep brain stimulation of the subthalamic region. Brain Stimul 5(3):369-377. doi:10.1016/j.brs.2011.05.002

6. Contarino MF, Lo Bour J, Verhagen R, Lourens MAJ, de Bie Rob M A, van den Munckhof P et al (2014) Directional steering: a novel approach to deep brain stimulation. Neurology 83(13):1163-1169. doi:10.1212/WNL.0000000000000823

7. Elble RJ, Pullman SL, Matsumoto JY, Raethjen J, Deuschl G, Tintner R (2006) Tremor amplitude is logarithmically related to 4- and 5-point tremor rating scales. Brain J Neurol 129(Pt 10):2660-2666. doi:10.1093/brain/awl190

8. Gantert C, Honerkamp J, Timmer J (1992) Analyzing the dynamics of hand tremor time series. Biol Cybern 66(6):479-484

9. Goetz CG, Tilley BC, Shaftman SR, Stebbins GT, Fahn S, Martinez-Martin P et al (2008) Movement disorder society-sponsored revision of the unified Parkinson's disease rating scale (MDS-UPDRS): scale presentation and clinimetric testing results. Mov Disord 23(15):2129-2170. doi:10.1002/mds.22340

10. Greenwald E, Masters MR, Thakor NV (2016) Implantable neurotechnologies: bidirectional neural interfaces-applications and VLSI circuit implementations. Med Biol Eng Comput 54(1):1-17. doi:10.1007/s11517-015-1429-x

11. Griffiths RI, Kotschet K, Arfon S, Xu ZM, Johnson W, Drago J et al (2012) Automated assessment of bradykinesia and dyskinesia in Parkinson's disease. J Parkinson's Dis 2(1):47-55. doi:10.3233/JPD-201211071

12. Hemm S, Wårdell K (2010) Stereotactic implantation of deep brain stimulation electrodes: a review of technical systems, methods and emerging tools. Med Biol Eng Comput 48(7):611-624. doi:10.1007/s11517-010-0633-y

13. Jankovic J, Frost JD (1981) Quantitative assessment of parkinsonian and essential tremor: clinical application of triaxial accelerometry. Neurology 31(10):1235. doi:10.1212/WNL.31.10.1235 
14. Journee HL, Postma AA, Staal MJ (2007) Intraoperative neurophysiological assessment of disabling symptoms in DBS surgery. Neurophysiol Clin Clin Neurophysiol 37(6):467-475. doi:10.1016/j.neucli.2007.10.006

15. Journée HL, Postma AA, Sun M, Staal MJ (2008) Detection of tremor bursts by a running second order moment function and analysis using interburst histograms. Med Eng Phys 30(1):75-83. doi:10.1016/j.medengphy.2006.12.005

16. Lang AE, Widner H (2002) Deep brain stimulation for Parkinson's disease: patient selection and evaluation. Mov Disord 17(S3):S94-S101. doi:10.1002/mds.10149

17. Lauk M, Timmer J, Guschlbauer B, Hellwig B, Lücking CH (2001) Variability of frequency and phase between antagonistic muscle pairs in pathological human tremors. Muscle Nerve 24(10):1365-1370

18. Mansur PHG, Cury LKP, Andrade AO, Pereira AA, Miotto GAA, Soares AB et al (2007) A review on techniques for tremor recording and quantification. Crit Rev Biomed Eng 35(5):343-362

19. Martens HCF, Toader E, Decré MMJ, Anderson DJ, Vetter R, Kipke DR et al (2011) Spatial steering of deep brain stimulation volumes using a novel lead design. Clin Neurophysiol 122(3):558-566. doi:10.1016/j.clinph.2010.07.026

20. Meigal AY, Rissanen SM, Tarvainen MP, Georgiadis SD, Karjalainen PA, Airaksinen O et al (2012) Linear and nonlinear tremor acceleration characteristics in patients with Parkinson's disease. Physiol Meas 33(3):395-412. doi:10.1088/0967-3334/33/3/395

21. Meldrum SJ, Watson BW (1970) Tremor recording in Parkinson's disease. Phys Med Biol 15(2):249254. doi:10.1088/0031-9155/15/2/302

22. Mera T, Vitek JL, Alberts JL, Giuffrida JP (2011) Kinematic optimization of deep brain stimulation across multiple motor symptoms in Parkinson's disease. J Neurosci Methods 198(2):280-286. doi:10.1016/j.jneumeth.2011.03.019

23. Moore GP, Ding L, Bronte-Stewart HM (2000) Concurrent Parkinson tremors. J Physiol 529(Pt 1):273-281

24. Morishita T, Foote KD, Wu SS, Jacobson CE, Rodriguez RL, Haq IU et al (2010) Brain penetration effects of microelectrodes and deep brain stimulation leads in ventral intermediate nucleus stimulation for essential tremor. J Neurosurg 112(3):491-496. doi:10.3171/2009.7.JNS09150

25. O'Suilleabhain PE, Matsumoto JY (1998) Time-frequency analysis of tremors. Brain J Neurol 121(Pt 11):2127-2134

26. Palmer JL, Coats MA, Roe CM, Hanko SM, Xiong C, Morris JC (2010) Unified Parkinson's disease rating scale-motor exam: inter-rater reliability of advanced practice nurse and neurologist assessments. $J$ Adv Nurs 66(6):1382-1387. doi:10.1111/j.1365-2648.2010.05313.x

27. Papapetropoulos S, Jagid JR, Sengun C, Singer C, Gallo BV (2008) Objective monitoring of tremor and bradykinesia during DBS surgery for Parkinson disease. Neurology 70(15):1244-1249. doi:10.1212/01.wnl.0000308936.27780.94

28. Papapetropoulos S, Katzen HL, Scanlon BK, Guevara A, Singer C, Levin BE (2010) Objective quantification of neuromotor symptoms in Parkinson's disease: implementation of a portable, computerized measurement tool. Parkinson's Dis 2010:760196. doi:10.4061/2010/760196 
29. Patel S, Lorincz K, Hughes R, Huggins N, Growdon J, Standaert D et al (2009) Monitoring motor fluctuations in patients with Parkinson's disease using wearable sensors. IEEE Trans Inf Technol Biomed Publ IEEE Eng Med Biol Soc 13(6):864-873. doi:10.1109/TITB.2009.2033471

30. Perera T, Yohanandan SAC, McDermott HJ (2015) A simple and inexpensive test-rig for evaluating the performance of motion sensors used in movement disorders research. Med Biol Eng Comput 54(23):333-339. doi:10.1007/s11517-015-1314-7

31. Pollo C, Kaelin-Lang A, Oertel MF, Stieglitz L, Taub E, Fuhr P et al (2014) Directional deep brain stimulation: an intraoperative double-blind pilot study. Brain $J$ Neurol 137(Pt 7):2015-2026. doi:10.1093/brain/awu102

32. Popović Maneski L, Jorgovanović N, Ilić V, Došen S, Keller T, Popović MB et al (2011) Electrical stimulation for the suppression of pathological tremor. Med Biol Eng Comput 49(10):1187-1193. doi:10.1007/s11517-011-0803-6

33. Post B, Merkus MP, de Bie Rob M A, de Haan Rob J, Speelman JD (2005) Unified Parkinson's disease rating scale motor examination: are ratings of nurses, residents in neurology, and movement disorders specialists interchangeable? Mov Disord 20(12):1577-1584. doi:10.1002/mds.20640

34. Pulliam CL, Heldman DA, Orcutt TH, Mera TO, Giuffrida JP, Vitek JL (2015) Motion sensor strategies for automated optimization of deep brain stimulation in Parkinson's disease. Parkinsonism Rel Disord 21(4):378-382. doi:10.1016/j.parkreldis.2015.01.018

35. Rissanen SM, Kankaanpää M, Meigal A, Tarvainen MP, Nuutinen J, Tarkka IM et al (2008) Surface EMG and acceleration signals in Parkinson's disease: feature extraction and cluster analysis. Med Biol Eng Comput 46(9):849-858. doi:10.1007/s11517-008-0369-0

36. Rissanen SM, Kankaanpaä M, Tarvainen MP, Novak V, Novak P, Hu K et al (2011) Analysis of EMG and acceleration signals for quantifying the effects of deep brain stimulation in Parkinson's disease. IEEE Trans Biomed Eng 58(9):2545-2553. doi:10.1109/TBME.2011.2159380

37. Riviere CN, Reich SG, Thakor NV (1997) Adaptive Fourier modeling for quantification of tremor. $J$ Neurosci Methods 74(1):77-87

38. Rocon E, Pons JL, Andrade AO, Nasuto SJ (2006) Application of EMD as a novel technique for the study of tremor time series. In: Conference proceedings: annual international conference of the IEEE engineering in medicine and biology society. IEEE engineering in medicine and biology society. Annual conference, Supplementary, pp 6533-6536. doi:10.1109/IEMBS.2006.260871

39. Sarem-Aslani A, Mullett K (2011) Industrial perspective on deep brain stimulation: history, current state, and future developments. Front Integr Neurosci. doi:10.3389/fnint.2011.00046

40. Senova S, Querlioz D, Thiriez C, Jedynak P, Jarraya B, Palfi S (2015) Using the accelerometers integrated in smartphones to evaluate essential tremor. Stereotact Funct Neurosurg 93(2):94-101. doi:10.1159/000369354

41. Shah A, Coste J, Lemaire J-J, Schkommodau E, Hemm-Ode S (2013) A method to quantitatively evaluate changes in tremor during deep brain stimulation surgery. In: 6th international IEEE/EMBS conference on neural engineering, pp 1202-1205. doi:10.1109/NER.2013.6696155

42. Shah AA, Coste J, Lemaire J-J, Ulla M, Schkommodau E, Hemm-Ode S (2014) Using acceleration sensors to quantify symptoms during deep brain stimulation surgery: poster presentations. Mov Disord 29(S1):30. doi:10.1002/mds. 25914 
43. Shamir RR, Eitan R, Sheffer S, Marmor-Levin O, Valsky D, Moshel S et al (2013). Intra-operative identification of the subthalamic nucleus motor zone using goniometers. In Hutchison D, Kanade T, Kittler J, Kleinberg JM, Mattern F, Mitchell JC et al (eds) Information processing in computer-assisted interventions, vol 7915. Lecture notes in computer science). Springer, Berlin, pp 21-29

44. Sowman PF, Türker KS (2005) Methods of time and frequency domain examination of physiological tremor in the human jaw. Hum Mov Sci 24(5-6):657-666. doi:10.1016/j.humov.2005.09.003

45. Spearman C (1904) The Proof and measurement of association between two things. Am J Psychol 15(1):72. doi:10.2307/1412159

46. Stacy M (2009) Medical treatment of Parkinson disease. Neurol Clin 27(3):605-631. doi:10.1016/j.ncl.2009.04.009

47. Sturman MM, Vaillancourt DE, Metman LV, Bakay RAE, Corcos DM (2004) Effects of subthalamic nucleus stimulation and medication on resting and postural tremor in Parkinson's disease. Brain J Neurol 127(Pt 9):2131-2143. doi:10.1093/brain/awh237

48. Tarvainen M, Ranta-aho P, Karjalainen P (2002) An advanced detrending method with application to HRV analysis. IEEE Trans Biomed Eng 49(2):172-175. doi:10.1109/10.979357

49. Veluvolu KC, Ang WT (2011) Estimation of physiological tremor from accelerometers for real-time applications. Sensors 11(12):3020-3036. doi:10.3390/s110303020

50. Wilcoxon F (1945) Individual comparisons by ranking methods. Biom Bull 1(6):80-83 